\title{
Personalized Neoantigen Peptide Vaccine iNeo-Vac-P01
}

National Cancer Institute

\section{Source}

National Cancer Institute. Personalized Neoantigen Peptide Vaccine iNeo-Vac-P01. NCI

Thesaurus. Code C162633.

A peptide-based, personalized cancer vaccine consisting of patient-specific mutated long peptides, which are immunogenic and unique to the patient's tumor, with potential immunomodulating and antineoplastic activities. Upon vaccination with the personalized neoantigen peptide vaccine iNeo-Vac-P01, the peptides stimulate the host immune system to mount a specific and potent cytotoxic T-lymphocyte $(C T L)$ response against tumor cells expressing the neoantigens, which results in tumor cell lysis. 